\title{
Avaliação dos sintomas emocionais e comportamentais em adolescentes obesos
}

\section{Emotional and behavioral symptoms evaluation in obese adolescents}

\author{
Andricy Silva Linhares Sarmento* \\ Especialização em Adolescência para Equipe Multidisciplinar pela Universidade \\ Federal de São Paulo - UNIFESP, São Paulo, SP, Brasil
}

\section{Teresa Helena Schoen-Ferreira**}

Doutora em Ciências Aplicadas à Pediatria pela Universidade Federal de São Paulo UNIFESP, São Paulo, SP, Brasil

\section{Élide Helena Medeiros***}

Professora adjunta da Universidade Federal de São Paulo - UNIFESP, São Paulo, SP, Brasil

\section{I sa de Pádua Cintra****}

Professora adjunta da Universidade Federal de São Paulo - UNIFESP, São Paulo, $\mathrm{SP}$, Brasil

\begin{abstract}
RESUMO
Investigou-se neste trabalho sintomas emocionais e comportamentais em adolescentes obesos. Participaram desse estudo 248 adolescentes de ambos os sexos, com idades entre 11 e 18 anos, sendo 54 obesos acompanhados em um ambulatório-escola e 194 eutróficos, estudantes de escolas da região. Os adolescentes responderam ao Young Self Report (YSR), que é um questionário para triagem de habilidades sociais e problemas de comportamento. Não houve diferença estatisticamente significante entre obesos e eutróficos em relação aos escores obtidos no instrumento, embora um quarto dos obesos tivessem apresentado problemas com o contato social e quase um terço comportamentos agressivos. Conclui-se que não se pode considerar os obesos como um grupo homogêneo em relação aos seus comportamentos ou saúde mental.
\end{abstract}

Palavras-chave: Obesidade, Avaliação psicológica, Adolescentes.

\begin{abstract}
The study investigated emotional and behavioral symptoms in 54 obese adolescents of both sex, aged 11 to 18 years old, attending the Centro de Atendimento e Apoio ao Adolescente (CAAA). Other 194 eutrophic teenagers, paired by gender and age, formed the control group. Two instruments were used in the investigation: Young Self Report (YSR) and a file of social demographic data, especially designed for this study. In relation to the study variables, no statistical differences between obese and eutrophic adolescents were observed although $25 \%$ of the obese
\end{abstract}


adolescents presented problems of social contact and almost 33\% showed aggressive behavior.

Keywords: Obesity, Psychological evaluation, Adolescents.

A adolescência é marcada por transformações físicas aceleradas, características da puberdade, diferentes do crescimento e desenvolvimento que ocorrem em ritmo constante na infância. Essas alterações são influenciadas por fatores hereditários, ambientais, nutricionais, psicológicos e sexuais. Além do aspecto físico, há também mudanças sociais, quando o adolescente começa a adquirir independência e responsabilidades, e mudanças psicológicas, como a diferenciação da capacidade cognitiva e adaptações de personalidade, constituindo uma parte da população com características fisiológicas e psicológicas específicas (GAMBARDELLA; FRUTUOSO; FRANCH, 1999).

Todas as transformações da adolescência têm efeito sobre o comportamento alimentar, influenciado por fatores internos como: auto-imagem, necessidades fisiológicas e saúde individual, valores, preferências e desenvolvimento psicossocial; e por fatores externos, como: hábitos familiares, amigos, valores e regras sociais e culturais, mídia, modismos, experiências e conhecimentos do indivíduo, nível socioeconômico e raça/etnia (FARTHING, 1991; NEUMARKSZTAINER; STORY; HANNAN ; CROLL, 2002).

Obesidade é a quantidade excessiva de gordura corpórea para um dado peso corpóreo (RODRIGUES; OLIVEIRA; CINTRA; FISBERG, 2005). O sobrepeso e a obesidade em crianças, adolescentes e adultos são problemas crescentes de saúde pública em países desenvolvidos (DUTRA; ARAÚJO; BERTOLDI, 2006). No Brasil, dados da Associação Brasileira para Estudo da Obesidade - Abeso mostram que aproximadamente $40 \%$ da população brasileira apresentam excesso de peso (FISBERG, 2004).

Entre as características psicológicas das crianças e dos adolescentes obesos são encontradas frequentemente a imaturidade, a infantilização, a dependência, a passividade, a baixa autoestima, a falta de iniciativa e a dificuldade de adiar gratificações. Observa-se que estas características estão relacionadas com alterações importantes na dinâmica psicossocial da criança obesa e com a performance intelectual (CAMPOS; FISBERG, 2004).

Santos e Cardoso (1999/2000) dizem que na fase da adolescência surgem interesses e expectativas diferentes. Os adolescentes obesos ficam mais incomodados, que quando crianças, com os problemas que ocorrem na compra de roupas, a preocupação com a aparência, e os apelidos que despertam sentimentos de mágoa e revolta. Sentemse feios e apresentam baixa autoestima. 
Quando a criança obesa atinge a adolescência, o estigma da obesidade já contribui para uma auto-imagem negativa, comportamento passivo e isolamento social que são reforçados pela pouca aceitação de si mesmo e do grupo de pares. Portanto, se não houver uma boa intervenção, todos estes aspectos serão incorporados à identidade adulta (MULLER, 2001). Estes estigmas alimentam o risco de desencadeamento de quadros psiquiátricos como depressão, transtornos ansiosos, abuso de álcool, drogas e transtornos alimentares (CORDAS; ASCENCIO, 2006).

Considerando que aspectos emocionais podem estar associados à obesidade, favorecendo muitas vezes o desenvolvimento de problemas psicológicos, como ansiedade, depressão e dificuldades comportamentais, é fundamental compreender 0 papel destes transtornos na etiologia ou mesmo como consequência da obesidade (LUIZ; GORAYEB; LIBERATORE JR.; DOMINGOS, 2005).

Os transtornos afetivos interferem na vida da criança e do adolescente, prejudicando de modo importante seu rendimento escolar e seu relacionamento familiar e social (FU I; CURATOLO; FRIEDRICH, 2000). Dobrow, Kamenetz e Devlin (2002) relatam vários estudos com índices significativamente elevados de depressão entre indivíduos obesos que procuram tratamento.

O estudo de Santos, Richardsands e Bleckley (2007) mostrou uma correlação positiva entre o Índice de Massa Corporal - IMC - e depressão em adolescentes de ambos os sexos e uma correlação negativa entre autoestima ou suporte social e o IMC. Em um estudo longitudinal realizado por Courteney, Gamboz e Johnson (2008), foi observado maior número de adolescentes obesos com sintomas de depressão e baixa autoestima nos dois momentos de coleta de dados. Entretanto, no segundo momento foi evidenciado que sintomas depressivos antecediam comportamentos problemáticos em relação à alimentação.

Sierra-Baigrie, Lemos-Giráldez e Fonseca-Pedrero (2009) observaram que adolescentes que apresentavam distorções no seu comportamento alimentar, também apresentavam significativamente mais comportamentos depressivos, problemas com o pensamento, problemas com o contato social e com a atenção e comportamentos ansiosos, que o grupo controle. Nesse estudo não houve diferença significativa no tocante aos comportamentos agressivos ou de transtorno de conduta.

Ansiedade é um sentimento vago e desagradável de medo, apreensão, caracterizado por tensão ou desconforto derivado de antecipação de perigo, de algo desconhecido ou estranho (SWEDO; LEONARD; ALLEN, 1994; ASBAHR, 2004). A ansiedade e o medo passam a ser reconhecidos como patológicos quando são exagerados, desproporcionais em relação ao estímulo, ou qualitativamente diversos do que se observa como norma naquela faixa etária, e 
interferem com a qualidade de vida, o conforto emocional ou $O$ desempenho diário do indivíduo (CASTILLO; RECONDO; ASBAHR; MANFRO, 2000). Os transtornos ansiosos são um dos quadros psiquiátricos mais comuns, tanto em crianças como em adultos, independente do Índice de Massa Corporal.

Ansiedade é um fator muito citado como presente na dinâmica da personalidade do individuo obeso (CATANEO; CARVALHO; GALINDO, 2005). Um estudo encontrou maiores índices de ansiedade, fobia social e ansiedade de separação entre obesos (VILA; ZIPPER; DABBAS; BERTRAND; ROBERT; RICOUR; MOUREN; SIMÉONI, 2004). Por outro lado, em um outro estudo, quanto à maturidade emocional, autoconceito, lócus de controle e ansiedade, não foram encontradas diferenças significantes entre adolescentes obesas e não-obesas (CATANEO; CARVALHO; GALINDO, 2005). Outro trabalho observou que, embora estivessem mais insatisfeitas com o corpo, sentimento que também apareceu entre os adolescentes não-obesos, os obesos identificaram em si características positivas (CARVALHO; CATANEO; GALINDO; MALFARÁ, 2005). Nguyen-Rodrigues, Chou, Unger e Spruijt-Metz (2008) observaram que problemas emocionais ou comportamentais independiam do IMC. Para eles é necessário outros fatores, como a presença de uma doença crônica, para desencadear estresse ou desordens alimentares, por exemplo.

Estudos diversos vêm sugerindo não haver diferença no funcionamento psicológico entre pessoas obesas e não-obesas, embora observa-se que obesidade pode desencadear ou agravar comorbidades psiquiátricas ou problemas emocionais, que devem ser considerados na avaliação ou manejo clínicos (NGUYEN-RODRIGUES; CHOU; UNGER; SPRUIJT-METZ, 2008; SARWER; THOMPSON; MITCHELL; RUBIN, 2008; SONG; FERNSTROM, 2008; KUNKEL; OLIVEIRA ; PERES, 2009).

As questões psicológicas que cercam a obesidade são muito importantes. Assim o objetivo deste estudo foi avaliar sintomas emocionais e comportamentais em adolescentes obesos. 0 conhecimento dos sintomas emocionais e comportamentais, ligados à obesidade em adolescentes é fundamental para permitir o desenvolvimento de ações voltadas ao controle desse crescente problema e ajudar profissionais na elaboração de intervenções mais eficazes.

\section{MÉTODO}

Participantes: Participaram desse estudo 248 adolescentes que não apresentavam problemas orgânicos evidentes nem alterações no desenvolvimento ou déficits sensoriais. Foram divididos em 2 grupos: o grupo de estudo e o grupo controle. 
Grupo de estudo: 54 adolescentes de ambos os sexos, com IMC $\geq$ 95, segundo critérios da Center for Disease Control and Prevention (2000) que eram acompanhados no Centro de Atendimento e Apoio ao Adolescente (CAAA), ou que estudavam em escolas da região atendida pelo Hospital São Paulo.

O CAAA é um serviço ambulatorial do Hospital São Paulo integrado ao Departamento de Pediatria da Universidade Federal de São Paulo (Unifesp), e atende adolescentes de 10 a 20 anos e conta com uma equipe multidisciplinar (médicos, psicólogos, nuticionistas, fonoaudiólogos, e outras especialidades). O ambulatório tem as mesmas características de um hospital-escola, apresentando trabalhos de ensino, extensão e pesquisa. Destaca-se como principal objetivo do CAAA dar assistência integral à saúde do adolescente, em seus aspectos orgânicos, psicoemocionais e sociais, visando prevenir problemas como os decorrentes da insegurança e baixa autoestima, para que possa atingir a idade adulta com saúde e prazer de viver (SCHOEN-FERREIRA; SILVA; FARIAS; SILVARES, 2002).

Grupo controle: 194 adolescentes pareados por sexo e idade com os adolescentes do grupo controle, considerados eutróficos, que eram acompanhados no Centro de Atendimento e Apoio ao Adolescente (CAAA), ou que estudavam em escolas da região atendida pelo Hospital São Paulo.

\section{Instrumentos}

Foram utilizados o Young Self Report - YSR (ACHENBACH; RESCORLA, 2001) e dados de avaliação nutricional, como as medidas de estatura e massa corporal dos adolescentes. A estatura foi analisada segundo o protocolo proposto por Gordon, Chumlea e Roche (1988). A massa corporal foi analisada segundo o protocolo proposto por Gordon et al. (1988). O IMC foi calculado por meio da equação apresentada por Quetelet: IMC = massa corporal $\div$ estatura $^{2}$, sendo que a unidade de medida da massa corporal é o quilograma e a da estatura é o metro (KUCZMARSKI; OGDEN; GUO; GRUMMER-STRAWN; FLEGAL; MEI; WEI; CRUTIN; ROCHE; JOHNSON, 2002).

Young Self Report é um inventário de comportamentos, autoaplicável, para jovens dos 11 aos 18 anos, derivado do Child Behavior Checklist $(\mathrm{CBCL})$. Foi estruturado para obter respostas do adolescente a respeito de suas próprias competências e problemas (ACHENBACH; RESCORLA, 2001).

O inventário é composto por duas partes, embora neste trabalho apresentemos apenas a segunda, que corresponde aos itens que avaliam problemas de comportamento. As respostas do participante devem referir-se somente aos últimos seis meses, contados a partir da data do preenchimento. O YSR foi estruturado para que 0 
adolescente gaste em torno de 15 minutos para responder ao instrumento. Os itens estão escritos na primeira pessoa do presente do indicativo.

A somatória dos escores obtidos na segunda parte permite ao avaliador traçar um perfil comportamental do adolescente, derivado da análise de oito agrupamentos de itens: I - Ansiedade/Depressão; II - Retraimento/Depressão; III - Queixas somáticas; IV - Problemas com o Contato Social; V - Problemas com o Pensamento; VI Problemas com a Atenção; VII - Violação de Regras; VIII Comportamento Agressivo. Os três primeiros agrupamentos formam a Escala de Internalização e os agrupamentos VII e VIII formam a Escala de Externalização. A soma de todos os itens forma a Escala Total. O YSR também permite agrupamentos orientados de acordo com o DSM IV TR (APA, 2002): Desordens Afetivas; Desordens de Ansiedade e Depressão; Problemas Somáticos; Transtorno do Déficit de Atenção e Hiperatividade; Transtorno Opositor e Transtorno de Conduta.

\section{Procedimentos}

O trabalho foi aprovado pelo Comitê de Ética em Pesquisa da Unifesp (CEP 1988/07) e autorizado pelo CAAA e duas escolas da região para que os alunos a partir da $7^{a}$ série $/ 8^{\circ}$ ano participassem. Todos os alunos que aceitaram participar e que seus pais ou responsáveis assinaram o Termo de Consentimento Livre e Esclarecido, foram convidados a responderem ao YSR de forma coletiva, em pequenos grupos, e, logo em seguida, fizeram a medida da estatura e massa corporal (realizadas por nutricionista), o procedimento de avaliação levou cerca de uma hora-aula. Foram informados ao estudante o objetivo e o sigilo do estudo.

Em seguida foram analisados os prontuários do CAAA, onde foram incluídos no estudo aqueles pacientes que tinham preenchido o YSR durante a primeira consulta na sala de espera (para minimizar 0 efeito da escolaridade, os adolescentes que cursavam até a $6^{a}$ série/70 ano respondiam 0 instrumento através de entrevista.), seguindo a rotina do setor e que tinham IMC $>95$. Os dados do YSR foram digitados no programa específico - ASEBA/ADM (ACHENBACH; RESCORLA, 2001).

\section{Tratamento estatístico}

Para análise estatística foram utilizados o Teste Qui-Quadrado para independência, que é um teste não-paramétrico utilizado para se verificar se duas variáveis e seus níveis possuem ou não uma dependência (associação) estatística; o Teste de Igualdade de duas Proporções, que é um teste não-paramétrico que compara se a proporção de respostas de duas determinadas variáveis e/ou seus níveis são estatisticamente significantes; e o Teste de Fisher foi 
utilizado para alguns casos, onde foi a frequência esperada foi menor que cinco observações.

\section{RESULTADOS}

\section{a) Quanto à descrição da amostra}

A amostra foi composta pelo dobro de meninas, tanto no grupo de estudo quanto no grupo de controle. A idade média dos eutróficos foi de 14,64 anos; e dos obesos $13,96(p=0,002)$. Em relação à altura, a média foi, em ambos os grupos, $1,64 \mathrm{~m}(p=0,836)$. Houve diferença estatisticamente significante em relação ao peso $(p<0,001)$ : o peso médio do grupo controle foi de $54,72 \mathrm{~kg}$ (DP 7,51), do grupo estudo $82,58 \mathrm{~kg}$ (DP 20,86). Também houve diferença estatisticamente significante $(p<0,001)$ em relação ao IMC: o do grupo controle foi de 20,26 (DP 1,98), do grupo estudo 30,83 kg (DP 7,38). Os sujeitos estudavam da $4^{\text {a }}$ série do Ensino Fundamental ao $3^{\mathrm{a}}$ ano do Ensino Médio, principalmente entre a $7^{\mathrm{a}}$ do ensino fundamental e o $3^{\circ}$ ano do ensino médio.

\section{b) Quanto aos resultados no YSR}

$\mathrm{Na}$ Tabela 1 podemos observar que não houve diferença estatisticamente significante entre obesos e eutróficos nos oito agrupamentos do YSR, refutando assim a hipótese onde os adolescentes obesos apresentariam escores mais altos nos oito agrupamentos do YSR.

Tabela 1: Distribuição da amostra considerada clínica, em porcentagem, nos oito agrupamentos do YSR, por IMC; percentil, nas três escalas do YSR.

\begin{tabular}{|c|c|c|c|}
\hline $\begin{array}{c}\text { Agrupamentos do } \\
\text { YSR }\end{array}$ & Grupo & Porcentagem & P-valor \\
\hline \multirow{2}{*}{$\begin{array}{l}\text { Ansiedade/ } \\
\text { retraimento }\end{array}$} & Eutróficos & 24,7 & \multirow{2}{*}{0,702} \\
\hline & Obesos & 22,2 & \\
\hline \multirow{2}{*}{$\begin{array}{l}\text { Isolamento/ } \\
\text { depressão }\end{array}$} & Eutróficos & 13,9 & \multirow{2}{*}{0,867} \\
\hline & Obesos & 14,8 & \\
\hline \multirow{2}{*}{ Problema somático } & Eutróficos & 12,4 & \multirow{2}{*}{0,636} \\
\hline & Obesos & 14,8 & \\
\hline \multirow{2}{*}{$\begin{array}{l}\text { Problema com o } \\
\text { contato social }\end{array}$} & Eutróficos & 17,5 & \multirow{2}{*}{0,167} \\
\hline & Obesos & 25,9 & \\
\hline \multirow{2}{*}{$\begin{array}{l}\text { Problemas com o } \\
\text { Pensamento }\end{array}$} & Eutróficos & 14,9 & \multirow{2}{*}{0,202} \\
\hline & Obesos & 9,3 & \\
\hline \multirow{2}{*}{$\begin{array}{l}\text { Problemas com } \\
\text { Atenção }\end{array}$} & Eutróficos & 21,1 & \multirow{2}{*}{0,674} \\
\hline & Obesos & 18,5 & \\
\hline \multirow{2}{*}{$\begin{array}{l}\text { Problemas com } \\
\text { Regras }\end{array}$} & Eutróficos & 10,3 & \multirow{2}{*}{0,355} \\
\hline & Obesos & 14,8 & \\
\hline \multirow{2}{*}{ Agressividade } & Eutróficos & 26,3 & \multirow{2}{*}{0,449} \\
\hline & Obesos & 31,5 & \\
\hline
\end{tabular}


Podemos observar na Tabela 2 que não houve diferença estatisticamente significante entre obesos e eutróficos nas três escalas do YSR, refutando a hipótese que dizia que os obesos apresentariam mais problemas de comportamento que os eutróricos; e refutando também a hipótese que dizia que os obesos, quando comparados consigo mesmos apresentariam mais problemas internalizantes.

Tabela 2: Distribuição da amostra considerada clínica, em porcentagem e percentil, nas três escalas do YSR.

\begin{tabular}{|c|c|c|c|}
\hline Escalas do YSR & Grupo & Porcentagem & P-valor \\
\hline \multirow{2}{*}{ Internalizante } & Eutróficos & 39,7 & \multirow{2}{*}{0,915} \\
\cline { 2 - 3 } & Obesos & 38,9 & 0 \\
\hline \multirow{2}{*}{ Externalizante } & Eutróficos & 35,1 & 0,131 \\
\cline { 2 - 4 } & Obesos & 46,3 & 0,401 \\
\hline $\begin{array}{c}\text { Total de } \\
\text { problemas }\end{array}$ & Eutróficos & 41,8 & 0 \\
\cline { 2 - 3 } & Obesos & 48,1 & \\
\hline
\end{tabular}

Observamos, na Tabela 3, que não houve diferença estatisticamente significante entre obesos e eutróficos nos seis agrupamentos do YSR baseados no DSM IV ${ }^{T R}$, refutando assim a quarta hipótese que dizia que haveria mais adolescentes obesos com problemas comportamentais que eutróficos.

Tabela 3: Distribuição da amostra considerada clínica no YSR, em porcentagem e percentil, nos seis agrupamentos baseados no DSM IV, por grupo.

\begin{tabular}{|c|c|c|c|}
\hline $\begin{array}{l}\text { Agrupamentos } \\
\text { baseados no DSM IV }\end{array}$ & Grupo & Porcentagem & P-valor \\
\hline \multirow{2}{*}{ Transtorno afetivo } & Eutróficos & 18,0 & \multirow{2}{*}{0,321} \\
\hline & Obesos & 24,1 & \\
\hline \multirow{2}{*}{ Transtorno ansiedade } & Eutróficos & 17,5 & \multirow{2}{*}{0,140} \\
\hline & Obesos & 9,3 & \\
\hline \multirow{2}{*}{ Transtorno somato } & Eutróficos & 10,3 & \multirow{2}{*}{0,580} \\
\hline & Obesos & 13,0 & \\
\hline \multirow{2}{*}{ TDAH } & Eutróficos & 17,5 & \multirow{2}{*}{0,437} \\
\hline & Obesos & 22,2 & \\
\hline \multirow{2}{*}{$\begin{array}{l}\text { Transtorno desafiante } \\
\text { de oposição }\end{array}$} & Eutróficos & 20,6 & \multirow{2}{*}{0,403} \\
\hline & Obesos & 25,9 & \\
\hline \multirow{2}{*}{$\begin{array}{l}\text { Transtorno de } \\
\text { conduta }\end{array}$} & Eutróficos & 14,4 & \multirow{2}{*}{0,784} \\
\hline & Obesos & 13,0 & \\
\hline
\end{tabular}




\section{DISCUSSÃO}

Neste estudo, a prevalência de obesidade na adolescência foi maior em meninas $(67 \%)$ que em meninos $(33 \%)$, resultados semelhantes são descritos por outros autores (NEUTZLING; TADDEI; RODRIGUES; SIGULEM, 2000; ABRANTES; LAMOUNIER; COLOSIMO, 2002), embora haja estudo com uma prevalência maior de obesidade associada ao sexo masculino (FERNANDES; CODOGNO; CARDOSO; RONQUE; FREITAS JR; OLIVEIRA, 2009). Match, Gerer e Ellgring (2003) observaram que as mulheres ingerem mais alimentos como mecanismo compensatório para questões emocionais.

Lembramos que neste estudo, a amostra de obesos foi por conveniência, ou seja, os adolescentes que procuraram um local para realizarem o tratamento da obesidade. Pode ser que a obesidade incomode mais as meninas que os meninos, desta forma, há uma maior procura de adolescentes do sexo feminino. Segundo Branco, Hilário e Cintra (2006), e Kakeshita e Almeida (2006), há uma maior preocupação por parte do sexo feminino em relação à imagem corporal. No estudo realizado por Medeiros e Chung (1999), mais adolescentes do sexo feminino estavam inscritas em um ambulatório médico, e mais adolescentes do sexo feminino se queixavam de obesidade. Para os meninos, a queixa era baixa estatura.

De forma convergente com os resultados obtidos em vários estudos que investigam os aspectos psicológicos de obesos, este trabalho não encontrou diferença estatisticamente significante entre obesos e eutróficos no que diz respeito as variáveis estudadas. Alguns discutem que nem todos os obesos têm sentimentos negativos sobre o seu corpo (STUNKARD; WADDEN, 1992; CATANEO; CARVALHO; GALINDO, 2005). Embora muitos autores relatem diferença estatística com relação aos aspectos emocionais (CSABI; TENUI; MOLNAR, 2000; VILA; ZIPPER; DABBAS; BERTRAND; ROBERT; RICOUR; MOUREN; SIMÉONI, 2004; LUIZ; GORAYEB; LIBERATORE JR; DOMINGOS, 2005). Podemos observar que os dados da literatura não são conclusivos em relação aos aspectos emocionais relacionados com a obesidade, o que, de modo algum retira a importância do profissional que trabalha com saúde mental em auxiliar os pacientes obesos a fazer escolhas de estilos de vida saudáveis que os levem a um maior bem-estar, físico, psicológico ou social (DOBROW; KAMENETZ; DEVLIN, 2002).

Os estudos parecem indicar que estar acima do peso deixa o adolescente mais vulnerável a comportamentos depressivos. Cabe lembrar que neste estudo, a amostra foi por conveniência. Pode ser que, os adolescentes que procuraram ajuda para emagrecer, tenham um perfil um pouco diferente, visto que se estivessem apresentando depressão, talvez não tivessem condições de fazer o movimento em busca de ajuda. 
Apesar de não ter sido encontrado diferença estatística, mais de um quarto dos obesos tiveram problemas com o contato social, ou seja, os adolescentes obesos sentem-se excluídos do seu grupo de pares, são alvo de piadinhas, apelidos e gozações entre os colegas, enfrentando um grande desgaste emocional, levando o adolescente ao isolamento e a ter problemas relacionados à amizade (SCHOENFERREIRA; GODOY; DÂMASO; FISBERG; AZNAR-FARIAS, 2005, MENEGHELLI; SCHOEN-FERREIRA; VEIGA-GODOY; CINTRA, 2007). Este resultado vai ao encontro de outros estudos, que descrevem que na adolescência surgem interesses e expectativas diferentes. Os adolescentes obesos ficam mais incomodados, que quando crianças, em relação à aparência, e os apelidos despertam sentimentos de mágoa e revolta (SANTOS; CARDOSO, 1999/2000). De acordo com Muller (2001), quando a criança obesa atinge a adolescência, 0 estigma da obesidade já contribui para uma auto-imagem negativa, comportamento passivo e isolamento social que são reforçados pela pouca aceitação de si mesmo e do grupo de pares.

Com relação ao Transtorno de Déficit de Atenção e Hiperatividade TDAH, podemos observar que houve uma maior porcentagem de obesos com características de TDAH quando comparados com os adolescentes eutróficos. De acordo com Desidério e Miyazaki (2007), uma das principais características do TDAH é a impulsividade, ou seja, falta de controle e dificuldade em adiar gratificações, este resultado assemelha-se as principais características psicológicas de crianças e de adolescentes obesos, descritas por Campos e Fisberg (2004). Seria interessante se pensar que, ao ser diagnosticada uma criança com TDAH, junto com as orientações para estudo, por exemplo, deveriam ser dadas orientações para uma alimentação saudável, a fim de prevenir a obesidade.

Em relação a variável somatização, não houve diferença estatística, porém foram observados mais obesos com problemas somáticos. Outros estudos também encontraram resultados semelhantes, como um realizado com índios adolescentes americanos, onde se verificou que a insatisfação corporal afeta o funcionamento social, os relacionamentos entre pares, autoestima, ansiedade, depressão e somatização em adolescentes (NEWMAN; SONTAG; SALVATO, 2006). É importante ressaltar que muitos dos adolescentes obesos que participaram deste estudo provinham de famílias com outros membros obesos. A família age como suporte emocional para seus membros, reforçando as vantagens da obesidade, fortalecendo alianças em prol do membro que está obeso e minimizando os efeitos negativos (SALES, 2006). A aceitação de um grupo social é um fator importante de proteção contra os riscos de desenvolver comportamentos psicopatológicos. A família provavelmente seja mais condescendente com os comportamentos do adolescente que levam a obesidade porque outros membros também se comportam da mesma 
maneira. A dinâmica familiar protege seus membros contra comportamentos psicopatológicos, visto que a obesidade é encarada como aceitação do amor familiar e proteção: a matriarca demonstra seus amor fornecendo comidas calóricas, outros membros aceitam este amor, ingerindo o alimento, ou solicitando algum em especial. 0 sentimento de inibição pode ser minimizado com a ingestão de alimentos calóricos (MACHT; GERER; ELLGRING 2003).

Os problemas do adolescente surgem em contato com outros adolescentes, na escola, por exemplo (FERRIANI; DIAS; SILVA; MARTINS, 2005). Daí a razão do agrupamento 'problemas sociais' apresentar um índice maior de participantes obesos na escala clínica. Entretanto, ao retornar ao lar, os efeitos destruidores são aplacados pela família acolhedora. A convivência com outros obesos pode favorecer 0 desenvolvimento de comportamentos psicologicamente sadios, evitando o desenvolvimento de psicopatologias. Em família, o adolescente obeso sente-se aceito.

Conclui-se que mais pesquisas sejam necessárias para elucidação dos motivos pelos quais alguns adolescentes são mais propensos que outros para problemas emocionais, e que não se simplifique as características do adolescente obeso. Uma avaliação de características comportamentais e emocionais do adolescente obeso é importante para uma melhor aderência a estilos de vida saudáveis.

\section{Referências Bibliográficas}

ABRANTES, M. M.; LAMOUNIER, J. A.; COLOSIMO, E. A. Prevalência de sobrepeso e obesidade em crianças e adolescentes das regiões Sudeste e Nordeste. Jornal de Pediatria, Rio de Janeiro, v. 78, n. 4, p. 335-340, jul/ago 2002.

ACHENBACH, T. M.; RESCORLA, L. A. Manual for the ASEBA School - Age Forms \& Profiles. Burlington, VT: University of Vermont, Research Center for Children, Youth \& Families. Burlington, Vermont: University of Vermont, Department of Psychiatry, 2001.

AMERICAN PSIQUIATRIC ASSOCIATION. Manual Diagnóstico e Estatístico de Desordens Mentais (DSM-TV-TR, 2002) - 5 aed. Porto Alegre: Artes Médicas, 2002.

ASBAHR, F. R. Transtornos ansiosos na infância e adolescência: aspectos clínicos e neurobiológicos. Jornal de Pediatria, Rio de Janeiro, v. 80, n. 2, p. 28-34, abril 2004.

BRANCO, L. M.; HILÁRIO, M. O. E. ; CINTRA, I. P. Percepção e satisfação corporal em adolescentes e a relação com seu estado nutricional. Revista de Psiquiatria Clínica, São Paulo, v. 33, n. 6, p. 292-296, nov/dez 2006.

CAMPOS, A. L. R.; FISBERG, M. Quociente de inteligência de crianças e adolescentes obesos pela escala WECHSLER. In: FISBERG, M. 
Atualização em obesidade na infância e adolescência. São Paulo: Atheneu, 2004.

CARVALHO, A. M. P.; CATANEO, C.; GALINDO, E. M. C.; MALFARA, C. T. Autoconceito e imagem corporal em crianças obesas. Paideia Ribeirão Preto, v. 15, n. 30, p. 131-139, jan/abr 2005.

CASTILHO, A. R. G. L.; RECONDO, R.; ASBAHR, F. R.; MANFRO, G. G. Transtornos de ansiedade. Revista Brasileira de Psiquiatria, São Paulo, v. 22, s. 2, p. 20-23, dez 2000.

CATANEO, C.; CARVALHO, A. M. P.; GALINDO, E. M. C. Obesidade e aspectos psicológicos: maturidade emocional, auto-conceito, lócus de controle e ansiedade. Psicologia: Reflexão e Crítica, Porto Alegre, v. 18, n. 1, p. 39-46, jan/abr 2005.

CORDAS, T. A.; ASCENCIO, R. F. R. Tratamento comportamental da obesidade. Einstein, São Paulo, v. 4, s. 1, p, S44-S48, 2006.

COURTNEY, E. A.; GAMBOZ, J.; JOHNSON, J. G. Problematic eating behaviors in adolescents with low self-esteem and elevated depressive symptoms. Eating Behaviors, Nova York, v. 9, n. 4, p. 408-414, dez 2008.

CSABI, G.; TENUI, T.; MOLNAR, D. Depressive symptoms among obese children. J ournal of Eating Weight Disorders, Milano, Itália, v. 5, n. 1, p. 43-45, mar 2000.

DESIDÉRIO, R. C. S.; MIYAZAKI, M. C. O. S. Transtornos de déficit de atenção/hiperatividade (TDAH): Orientações para a família. Revista Semestral da Associação Brasileira de Psicologia Escolar e Educacional, São Paulo, v. 11, n. 1, p. 165-178, jan/jun 2007.

DOBROW, I. J.; KAMENETZ, C.; DEVLIN, M. J. Aspectos psiquiátricos da obesidade. Revista Brasileira de Psiquiatria, v. 24, p. 63-67, 2002.

DUTRA, C. L.; ARAUJO, C. L.; BERTOLDI, A. D. Prevalence of overweight in adolescents: a population-based study in a southern Brazilian city. Cadernos Saúde Pública, Rio de Janeiro, v. 22, n. 1, p. 151-162, jan 2006.

FARTHING, M. C. Current eating patterns of adolescents in the United States. Nutrition Today, Baltimore, EUA, v. 26, n. 2, p. 35-39, abr 1991.

FERNANDES, R. A.; CODOGNO, J. S.; CARDOSO, J. R.; RONQUE, E. R. V.; FREITAS JR, I. F.; OLIVEIRA, A. R. Fatores associados ao excesso de peso entre adolescentes de diferentes redes de ensino do município de Presidente Prudente, São Paulo. Revista Brasileira de Saúde Materno Infantil, Recife, v. 9, n. 4, p. 443-449, nov/dez 2009.

FERRIANI, M. G. C.; DIAS, T. S.; SILVA, K. Z. ; MARTINS, C. S. Autoimagem corporal de adolescentes atendidos em um programa multidisciplinar de assistência ao adolescente obeso. Revista Brasileira de Saúde Materno Infantil, Recife, v. 5, n. 1, p. 27-33, jan/mar 2005. 
FISBERG, M. Atualização em obesidade na infância e adolescência. São Paulo: Atheneu, 2004.

FU I, L.; CURATOLO, E.; FRIEDRICH, S. Transtornos afetivos. Revista Brasileira de Psiquiatria, São Paulo, v. 22, s. 2, p. 24-27, dez 2000.

GAMBARDELlA, A. M. D.; FRUTUOSO, M. F. P. ; FRANCH, C. Prática alimentar de adolescentes. Revista de Nutrição, Campinas, v. 12, n. 1, p. 55-63, jan/abr 1999.

GORDON, C. C.; CHUMLEA, W. C.; ROCHE, A. F. Stature, Recumbent LENGTH, AND WEIGHT. In: LOHMAN, T. G., ROCHE, A. F.; MARTORELL, R. Anthropometric standardization reference manual. Champaign (IL): Human Kinetics Books, p. 3-8, 1998.

KAKESHITA, I. S.; ALMEIDA, S. S. Relação entre índice de massa corporal e a percepção da auto-imagem em universitários. Revista de Saúde Pública, São Paulo, v. 40, n. 3, p. 497-504. Jun 2006.

KUCZMARSKI, R. J.; OGDEN, C. L.; GUO, S. S.; GRUMMER-STRAWN, L. M.; FLEGAL, K. M.; MEI, Z.; WEI, R.; CRUTIN, L. R.; ROCHE, A. F.; JOHNSON, C. L. 2000 CDC growth charts for the United States: methods and development t. Vital and Health Statistics, v. 11, n. 246, p. 1190, 2002.

KUNKEL, N.; OLIVEIRA, W. F.; PERES, M. A. Excesso de peso e qualidade de vida relacionada à saúde em adolescentes de Florianópolis, SC. Revista de Saúde Pública, São Paulo, v. 43, n. 2, p. 226-235, fev 2009.

LUIZ, A. M. A. G.; GORAYEB, R.; LIBERATORE JR, R. D. R. L.; DOMINGOS, N. A. M. Depressão, ansiedade, competência social e problemas comportamentais em crianças obesas. Estudos de Psicologia, Natal, v. 10, p. 3, p. 371-375, set/dez 2005.

MACHT, M.; GERER, J.; ELLGRING, H. Emotions in overweight and normal-weight women immediately after eating foods differing in energy. Physiology \& Behavior, v. 80, n. 2-3, p. 367-374, 2003. MEDEIROS, E. H. G. R.; CHUNG, S. S. O adolescente do Centro de Atendimento e Apoio ao Adolescente (CAAA). UNIFESP-EPM. Brazilian Pediatric News, v. 1, n. 4, dez 1999. Disponível em: $<$ http://www.brazilpednews.org.br/dezem99>. Acesso em: 30 Ago. 2008.

MENEGHELLI, N. SCHOEN-FERREIRA, T. H.; VEIGA-GODOY, Y. E.; CINTRA, I. P. Sou motivo de Gozação. In: X Congresso Brasileiro de Adolescência, 2007, Foz do Iguaçu. Adolescer na diversidade, 2007.

MULLER, R. C. L. Obesidade na adolescência. Pediatria Moderna, São Paulo, v. 38, n. 1, p. 45-48, jan/fev 2001. Disponível em: <http://www.cibersaude.com.br/revistas.asp?fase=r003\&id_materia $=1413>$. Acesso em: 16 Set. 2007.

NEUMARK-SZTAINER, D.; STORY, M.; HANNAN, P. J.; CROLL, J. Overweight Status and Eating Patterns Among Adolescents: Where Do Youths Stand in Comparison With the Healthy People 2010 
Objectives? American Journal of Public Health, Washington EUA, v. 92, n. 5, p. 844-851, maio 2002.

NEUTZLING, M. B.; TADDEI, J. A. A. C.; RODRIGUES, E. M.; SIGULEM, D. M. Overweight and obesity in Brazilian adolescents. Journal of International Obesity Metabolism Didorders, Londres, Ing, v. 24, n. 7, p. 869-874, jul 2000.

NEWMAN, L. D.; SONTAG, M. L.; SALVATO, R. Psychosocial aspects of body mass and body image among rural american indian adolescents. J ournal of Youth and adolescence. Nova York - EUA, v. 35, n. 2, p. 265-275, fev 2006.

NGUYEN-RODRIGUEZ, S. T.; CHOU, C. P.; UNGER, J. B.; SPRUIJTMETZ, D. BMI as a moderator of perceived stress and emotional eating in adolescents. Eating Behaviors, Nova York,- EUA, v. 9, n. 2, p. 238-246, set 2008.

SALES, C. A. I nfluência dos padrões interacionais da família na obesidade do adolescente: um estudo qualitativo com o Modelo Familiar FIRO. Dissertação (Mestrado) - Pontifícia Universidade Católica de São Paulo. São Paulo, 2006. Disponível em: http://www.sapientia.pucsp.br//tde busca/arquivo.php?codArquivo= 4646. Acesso em: 29 Ago. 2010.

SANTOS, E. C.; CARDOSO, A. L. Obesidade exógena infantil: abordagem social de uma problemática multifatorial. Serviço Social Hospitalar, v. 6/7, p. 29-33, 1999/2000.

SANTOS, M.; RICHARDSAND, C. S.; BLECKLEY, M. K. Comorbidity between depression and disordered eating in adolescents. Eating Behaviors, Nova York - EUA, v. 8, n. 4, p. 440-449, abr 2007.

SARWER, D. B.; THOMPSON, J. K.; MITCHELL, J. E.; RUBIN, J. P. Psychological considerations of the bariatric surgery patient undergoing body contouring surgery. Plastic and Reconstructive Surgery, Dallas - EUA, v. 121, n. 6, p. 423e-434e, jun 2008.

SCHOEN-FERREIRA, T. H.; SILVA, D. A.; FARIAS, M. A.; SILVARES, E. F. M. Perfil e principais queixas dos clientes encaminhados ao Centro de Atendimento e Apoio Psicológico ao Adolescente (CAAA) UNIFESP/EPM. Psicologia em estudo, Maringá, v. 7, n. 2, p. 73-82, mai/ago 2002.

SIERRA-BAIGRIE, S.; LEMOS-GIRÁLDEZ, S.; FONSECA-PEDRERO, E. Binge eating in adolescents: Its relation to behavioural problems and family meal patterns. Eating Behaviors, Nova York - EUA, v. 10, n. 1, p. 22-28, jan 2009.

SONG, A.; FERNSTROM, M. H. Nutritional and psychological considerations after bariatric surgery. Aesthetic Surgery Journal, v. 28, n. 2, p. 195-199, mar/abr 2008.

STUNKARD, A. J.; WADDEN, T. A. Psychological aspects, of severe obesity. The American Journal of Clinical Nutrition, v. 55, p. 524-532, 1992. 
SWEDO, S. E.; LEONARD, H. L; ALLEN, A. J. New developments in childhood affective and anxiety disorders. Current. Current Problems in Pediatrics, New Jersey - EUA, v. 24, n. 1, p. 12-38, jan 1994.

VILA, G.; ZIPPER, E.; DABBAS, M.; BERTRAND, C.; ROBERT, J. J.; RICOUR, C.; MOUREN; SIMÉONI, M. C. Mental disorders in obese children and adolescents. Psychosomatic Medicine, Palo Alto EUA, v. 66, n.3, p. 387-394, mar 2004.

\section{Endereço para correspondência}

Andricy Silva Linhares Sarmento

Universidade Federal de São Paulo, Centro de Atendimento e Apoio ao Adolescente - CAAA, Rua Botucatu, 715, Vila Clementino, São Paulo - SP, Brasil

Endereço eletrônico: andricy@msn.com

Teresa Helena Schoen-Ferreira

Universidade Federal de São Paulo, Centro de Atendimento e Apoio ao Adolescente - CAAA, Rua Botucatu, 715, Vila Clementino, São Paulo - SP, Brasil

Endereço eletrônico: rpetrass@uol.com.br

Elide Helena Medeiros

Universidade Federal de São Paulo, Centro de Atendimento e Apoio ao Adolescente - CAAA, Rua Botucatu, 715, Vila Clementino, São Paulo - SP, Brasil

Endereço eletrônico: ehmedeiros@uol.com.br

Isa de Pádua Cintra

Universidade Federal de São Paulo, Centro de Atendimento e Apoio ao Adolescente - CAAA, Rua Botucatu, 715, Vila Clementino, São Paulo - SP, Brasil

Endereço eletrônico: ipcintra@uol.com.br

Recebido em: 27/10/2009

Aceito para publicação em: 02/03/2010

Acompanhamento do processo editorial: Rita Maria Manso de Barros

\section{Notas}

*Psicóloga;

**Psicóloga; responsável pelo serviço de Psicologia no Centro de Atendimento e Apoio ao Adolescente, CAAA, do Departamento de Pediatria da Universidade Federal de São Paulo - UNIFESP

***Médica Pediatra; doutorado em Ciências pela Universidade Federal de São Paulo

- UNIFESP

****Nutricionista; mestrado em Ciências Biológicas pela UFMG; doutorado em Nutrição pela Universidade Federal de São Paulo - UNIFESP; Coordenadora do Curso de Especialização em Adolescência para Equipe Multidisciplinar da UNIFESP. 